\title{
Performed Substance Administration Dose Description
}

National Cancer Institute

\section{Source}

National Cancer Institute. Performed Substance Administration Dose Description. NCI

Thesaurus. Code C93972.

The textual representation of dosing amounts or a range of dosing information used in a substance administration. 